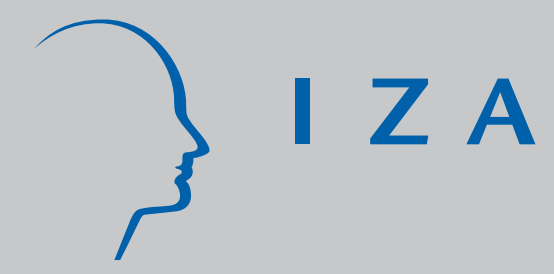

IZA DP No. 2393

The Macroeconomics of Targeting: The Case of an Enduring Epidemic

Clive Bell

Hans Gersbach

October 2006 


\title{
The Macroeconomics of Targeting: The Case of an Enduring Epidemic
}

\author{
Clive Bell \\ University of Heidelberg
}

Hans Gersbach

ETH Zurich and IZA Bonn

Discussion Paper No. 2393

October 2006

\author{
IZA \\ P.O. Box 7240 \\ 53072 Bonn \\ Germany \\ Phone: +49-228-3894-0 \\ Fax: +49-228-3894-180 \\ E-mail: iza@iza.org
}

\begin{abstract}
Any opinions expressed here are those of the author(s) and not those of the institute. Research disseminated by IZA may include views on policy, but the institute itself takes no institutional policy positions.

The Institute for the Study of Labor (IZA) in Bonn is a local and virtual international research center and a place of communication between science, politics and business. IZA is an independent nonprofit company supported by Deutsche Post World Net. The center is associated with the University of Bonn and offers a stimulating research environment through its research networks, research support, and visitors and doctoral programs. IZA engages in (i) original and internationally competitive research in all fields of labor economics, (ii) development of policy concepts, and (iii) dissemination of research results and concepts to the interested public.
\end{abstract}

IZA Discussion Papers often represent preliminary work and are circulated to encourage discussion. Citation of such a paper should account for its provisional character. A revised version may be available directly from the author. 
IZA Discussion Paper No. 2393

October 2006

\section{ABSTRACT}

\section{The Macroeconomics of Targeting: The Case of an Enduring Epidemic}

What is the right balance among policy interventions in order to ensure economic growth over the long run when an epidemic causes heavy mortality among young adults? We argue that, in general, policies to combat the disease and promote education must be concentrated, in certain ways, on some subgroups of society, at first to the partial exclusion of others. This concentration involves what we term the macroeconomics of targeting. The central comparison is then between programs under which supported families enjoy the benefits of spending on health and education simultaneously (DT), and those under which the benefits in these two domains are sequenced (ST). When levels of human capital are uniformly low at the outbreak, DT is superior to ST if the subsequent mortality rate exceeds some threshold value. Outside aid makes DT more attractive; but DT restricts support to fewer families initially and so increases inequality.

JEL Classification: E62, H20, I10, I20, O11

Keywords: epidemic diseases, HIVIAIDS, poverty traps, macroeconomics of targeting, education support, health policies, single and double targeting

Corresponding author:

Hans Gersbach

ETH Zurich

Center of Economic Research (CER-ETH)

ZUE D9

Zuerichbergstrasse 18

8092 Zuerich

Switzerland

E-mail: hgersbach@ethz.ch

\footnotetext{
*We thank Bernhard Pachl, Martin Scheffel, Ramona Bruhns, Lars Siemers and participants in several seminars for their valuable comments and suggestions.
} 


\section{Introduction}

Heavy mortality among young adults is a formidable obstacle to long-term economic growth. The investments in them are destroyed before they have had much time to pay off, and the transmission of knowledge and abilities from one generation to the next is weakened, perhaps fatally. A widespread epidemic of AIDS, which will carry off large numbers of young adults over a long stretch of time, poses a very real danger. Bell, Devarajan and Gersbach (2003), for example, show that the AIDS epidemic in South Africa may seriously hinder growth in that country, and that the threat of an economic collapse cannot be ruled out.

The object of this paper is to analyze how this threat can be best averted by interventions in the domains of health and education. The instruments available for this purpose are (i) measures to contain the disease and treat the infected, (ii) helping needy families and orphans, in the form of income-support or subsidies contingent on school attendance, and (iii) taxes to finance the expenditure program. The central policy problem is to find the right balance among these interventions in order to ensure economic growth over the long run without the emergence of excessive inequality.

We employ the overlapping generations (OLG) model model of Bell, Devarajan and Gersbach (2003), in which human capital formation is influenced by premature adult mortality in a disease-ridden environment. Parents have preferences over current consumption and the level of human capital attained by their children. The decision regarding how much to invest in education is influenced by the level of premature adult mortality in two ways: first, a family's lifetime income depends on the adults' health status, and second, the expected pay-off depends on the level of premature mortality among the children themselves when they attain adulthood, as subjectively estimated by their parents. The outbreak of an epidemic such as AIDS leads to an increase in such mortality, and if the prevalence of the disease becomes sufficiently high, a progressive collapse of human capital and productivity may follow - if the economy was already growing. If, however, levels of human capital were uniformly low, the problems of overcoming this condition of backwardness will be exacerbated. 
The rise in mortality can be checked, to some extent at least, by spending on measures to combat the epidemic, and its effects can be mitigated by providing support to those who fall sick and their families. By killing off mainly young adults, however, AIDS also undermines the tax base. As a result, the state's finances will come under increasing pressure from both sides, even as the demand for expenditures on the needy continues to grow.

In this dismal state of affairs, policy-making involves tough and ethically contentious choices. In examining them, we make the central assumption that fiscal resources are insufficient to support all needy families, a sadly valid assumption in the context of HIV/AIDS in most developing countries. We therefore investigate how public spending should be allocated between education and health, and whether and how spending should be concentrated on subgroups within the population, when the goal is to ensure long-term economic growth. Given this goal, the possible need for selective concentration involves what we term the macroeconomics of targeting. Under the resulting policies, one subgroup of needy families may receive support, while others with the same needs receive nothing. We distinguish further between what we call double-domain targeting, that is, simultaneous intervention in the domains of both health and education in the first period following the outbreak, and singledomain targeting, that is, intervention to promote education in the first period, followed by measures to promote health in the next period. Under double-domain targeting (henceforth DT), some needy families receive both the benefits of spending on health and transfers to promote education. Under single-domain targeting (henceforth ST), families receive educational support at the start, and the benefits of spending on health later.

Our main results are as follows. Our first claim is that, apart from treating and caring for the infected, policies to combat the disease and support education must be concentrated on subgroups of the society in order to ensure that human capital and per capita income increase at the highest possible rate. Spreading support evenly across the whole society will not necessarily help to combat the disease, nor will it foster human capital accumulation. Second, we characterize the conditions under which DT is preferred to ST. As a rule, we establish that DT is superior to ST if the mortality rate exceeds some threshold value. Moreover, 
outside aid makes DT more attractive relative to ST. The intuition is that, in the presence of high prevalence rates, a targeted group of needy families must be protected against infection while receiving sufficient educational support in order to enable their offspring to avoid poverty in adulthood. The dramatic implication of such a DT-policy is that the share of families receiving support is small, and thus leads to higher inequality, at least temporarily.

There is a growing literature on the long-run economic consequences of AIDS, many aspects of which are discussed in various contributions in Haacker (2004). The estimates of the size of its adverse effects diverge quite widely, for reasons set out briefly in Bell, Devarajan and Gersbach (2006). Some other contributions that also focus on human capital (Corrigan, Glomm and Méndez, 2004 and 2005; McDonald and Roberts, 2004) suggest that AIDS has large adverse effects on the growth rate of per capita income. In contrast, Young (2005), who employs a Solovian framework, arrives at the conclusion that these effects are small, if not actually positive. Such controversy aside, the present paper is complementary to a large literature on the microeconomic and public finance aspects of targeting in anti-poverty programs (see, for example, the discussion in Galasso and Ravallion [2005]), inasmuch as it deals with the macroeconomic aspects of targeting education and health policy.

The paper is organized as follows. In section 2, we lay out the model and discuss its dynamical behavior. This includes the possibility that a sudden rise in premature adult mortality will set off a progressive slide into a poverty trap, and so introduces the policy problem in section 3. The goal here is to bring about long-term economic growth in a society which is in a state of general economic backwardness, with low levels of human capital, when the epidemic breaks out, this being the most challenging constellation of initial conditions. The policy instruments are discussed in detail, drawing a careful distinction between spending on health to produce a private as opposed to a public good. We assume that restricted lump-sum taxes are possible, and that subsidies can be made conditional on a household's state. In section 4, we compare ST and DT, a comparison which leads to our main results. Section 5 provides a concluding discussion. 


\section{The Model}

We employ the OLG model of Bell, Devarajan and Gersbach (2003), which we summarize in this section. There are two periods of life, childhood and adulthood. Each generation consists of a continuum of households, which has measure $N_{t}$ at the start of period $t$ when the children raised in period $t-1$ have just entered adulthood. The initial population of households is normalized to $N_{0}=1$, and is thus identical to the interval $[0,1]$. On becoming adults, individuals form unions, start families and have children. Each couple has $n_{t}$ children, ${ }^{1}$ so that $N_{t+1}=N_{t} \cdot n_{t} / 2$. Shortly thereafter, the adults learn whether they will die prematurely as a consequence of HIV/AIDS, and so leave their children as half- or full orphans. Early in each generation of adults, therefore, all nuclear families are sorted into one of the following four categories: both parents survive into old age, the father dies prematurely, the mother dies prematurely, both parents die prematurely. These states are denoted by $s_{t} \in S_{t}:=\{1,2,3,4\}$, respectively. The probability that a family formed at the start of period $t$ lands in category $s_{t}$ is denoted by $\pi_{t}\left(s_{t}\right) .^{2}$ Once their states have been revealed, families make their decisions accordingly, as will be described below.

Consider a family at the start of period $t$. We assume assortative mating and denote the father's and mother's endowments of human capital by $\lambda_{t}$. When the family is revealed to be in state $s_{t}$, the adults' total human capital is therefore given by

$$
\Lambda_{t}(1)=2 \lambda_{t}, \Lambda_{t}(2)=\Lambda_{t}(3)=\lambda_{t}, \Lambda_{t}(4)=0
$$

Human capital is assumed to be formed by a process of child-rearing combined with formal education. In the course of rearing their children, parents give them a certain capacity to build human capital for adulthood, a capacity which itself depends on the parents' own human capital. This gift will be of little use, however, unless it is complemented by at least some formal education. Let the proportion of childhood devoted to education be denoted by

\footnotetext{
${ }^{1}$ We assume that fertility is exogenous. A three-generation variant of the model with endogenous fertility and support from children in old age can be found in Bruhns (2004).

${ }^{2}$ Assuming an appropriate version of the law of large numbers for a continuum, this is also the fraction of all families in that state after all premature adult deaths have occurred.
} 
$e_{t} \in[0,1]$, the residual being allocated to work, and for simplicity, let all the children in a family be treated in the same way. The human capital attained by each of the children on reaching adulthood is assumed to depend on their education and their parents' human capital as follows:

$$
\lambda_{t+1}= \begin{cases}z\left(s_{t}\right) f\left(e_{t}\right) \Lambda_{t}\left(s_{t}\right)+1, & s_{t}=1,2,3 \\ \xi \quad(\xi \leq 1) & s_{t}=4\end{cases}
$$

where the term $z\left(s_{t}\right)$ represents the strength with which capacity is transmitted across generations. For simplicity, we assume that the father's and mother's contributions are perfect substitutes, so that $z(2)=z(3)>z(1)=z(2) / 2=z(3) / 2$. Thus, the upper branch of (2) can be rewritten as

$$
\lambda_{t+1}=\left(3-s_{t}\right) z\left(s_{t}\right) f\left(e_{t}\right) \lambda_{t}+1, \quad s_{t}=1,2
$$

since both types of single-parent families are identical in this respect. This permits one to drop the state $s_{t}=3$ in much of the following. The function $f(\cdot)$ represents the educational technology - translating time spent on education into learning. $f(\cdot)$ is assumed to be strictly increasing and differentiable, with $f(0)=0$. Observe that eq. (2) and $f(0)=0$ imply that children who do not attend school at all attain, as adults, only some basic level of human capital, which has been normalized to unity. A whole society of such adults will be said to be in a state of economic backwardness.

According to the lower branch of (2), there is a miserable outcome for full orphans. Being left to their own devices, they go through childhood uneducated, to attain human capital $\xi$ $(\leq 1)$ in adulthood. For convenience, we set $\xi=1$.

Output takes the form of an aggregate consumption good. We assume its level is proportional to inputs of labor measured in efficiency units. A natural normalization is that an adult who possesses human capital in the amount $\lambda_{t}$ is endowed with $\lambda_{t}$ efficiency units of labor, which he or she supplies completely inelastically. Let a child supply $\left(1-e_{t}\right) \gamma$ efficiency units of labor when the child works $1-e_{t}$ units of time, where $\gamma \in(0,1)$, i.e., a full-time working child is less productive than an uneducated adult. A family with $n_{t}$ children therefore has a 
total income in state $s_{t}$ of

$$
y_{t}\left(s_{t}\right)=\alpha\left[\Lambda_{t}\left(s_{t}\right)+n_{t}\left(1-e_{t}\right) \gamma\right], \quad s_{t}=1,2,3
$$

where the scalar $\alpha(>0)$ denotes the productivity of human capital, measured in units of output.

\subsection{The Household's Behavior}

It is assumed that all allocative decisions lie in the parents' hands, as long as they are alive. We rule out any bequests at death, so that the whole current income, as given by (4), is consumed. Within the family, let the husband and wife enjoy equality as partners, and let each child obtain a fraction $\beta \in(0,1)$ of an adult's consumption if at least one adult survives. Full orphans $\left(s_{t}=4\right)$ do not attend school, and consume what they produce as child laborers. The household's budget constraint is therefore written as

$$
\left[\left(3-s_{t}\right)+n_{t} \beta\right] c_{t}+\alpha n_{t} \gamma e_{t} \leq \alpha\left[\left(3-s_{t}\right) \lambda_{t}+n_{t} \gamma\right], \quad s_{t}=1,2
$$

where $c_{t}$ is the level of each adult's consumption. The expression on the LHS represents the costs of consumption and the opportunity costs of the children's schooling. The expression on the RHS is the family's so-called full income ${ }^{3}$ in state $s_{t}=1,2$. Observe that singleparent households not only have lower levels of full income than their otherwise identical two-parent counterparts, but that they also face a higher relative price of education, which is defined as $\alpha n_{t} \gamma /\left[\left(3-s_{t}\right)+n_{t} \beta\right]$.

Couples have children while they are young until some exogenously fixed number have survived infancy, a target that may vary from period to period. With $n_{t}$ thus fixed, the adults wait until the state of the family becomes known, and the survivor(s) then choose some feasible bundle $\left(c_{t}, e_{t}\right)$ subject to (5).

\footnotetext{
${ }^{3}$ A household's full income is the scalar product of its endowment vector and the vector of market prices. Here, output is taken as the numéraire.
} 
Parents are assumed to have preferences over their own current consumption and the human capital attained by their children in adulthood, taking into account the fact that an investment in a child's education will be wholly wasted if that child dies prematurely in adulthood. Let mothers and fathers have identical preferences, and for two-parent households, let there be no 'joint' aspect to the consumption of the bundle $\left(c_{t}, e_{t}\right)$ : each surviving adult derives (expected) utility from the pair chosen in this way, and these utilities are then added up within the family. In effect, whereas $c_{t}$ is a private good, the human capital of the children in adulthood is a public good within the marriage.

Since all the children attain $\lambda_{t+1}$, the only form of uncertainty at the time of decision surrounds the number who will die prematurely as adults. Denote by $\pi_{t+1}^{e}\left(s_{t+1}\right)$ the parents' subjective probability that a child will find itself in state $s_{t+1}$ in period $t+1$, with $\sum_{s_{t+1}=1}^{4} \pi_{t+1}^{e}\left(s_{t+1}\right)=1$. Throughout the paper, we assume perfect foresight from $t=0$ onwards, so that $\pi_{t+1}^{e}\left(s_{t+1}\right)=\pi_{t+1}\left(s_{t+1}\right)$. Denoting by $p_{t}$ the probability that an adult will survive into old age, and noting that we have implicitly assumed that there are equal numbers of male and female children, we obtain

$$
p_{t} \equiv\left[1+\pi_{t}(1)-\pi_{t}(4)\right] / 2
$$

The expected number of children born in period $t$ who will survive into old age in period $t+1$ is $p_{t+1} n_{t}$.

Let preferences be separable, with representation

$$
E U_{t}\left(s_{t}\right)=\left(3-s_{t}\right)\left[u\left(c_{t}\right)+n_{t} p_{t+1} v\left(\lambda_{t+1}\right)\right], \quad s_{t}=1,2
$$

Observe that the contribution $v\left(\lambda_{t+1}\right)$ counts only when a grown child does not suffer an untimely death. The sub-utility functions $u(\cdot)$ and $v(\cdot)$ are assumed to be increasing, continuous, concave and twice-differentiable. The argument $\lambda_{t+1}$ is given by the upper branch of (2). Observing that $p_{t+1}=1$ (and $\pi_{t+1}(1)=1$ ) if there is no premature adult mortality, and that $p_{t+1}<1$ otherwise, it is seen that a reduction in $p_{t+1}$ effectively entails a weaker preference for the children's education. It will be convenient to rewrite (7) as

$$
E U_{t}\left(s_{t}\right)=\left(3-s_{t}\right)\left[u\left(c_{t}\right)+n_{t} p_{t+1} v\left(z\left(s_{t}\right) f\left(e_{t}\right) \Lambda_{t}\left(s_{t}\right)+1\right)\right], \quad s_{t}=1,2
$$


A family in state $s_{t}(=1,2)$ in period $t$ solves the following problem:

$$
\max _{\left[c_{t}\left(s_{t}\right), e_{t}\left(s_{t}\right)\right]} E U_{t}\left(s_{t}\right) \quad \text { s.t. }(5), c_{t} \geq 0, e_{t} \in[0,1]
$$

Let $\left[c_{t}^{0}\left(s_{t}\right), e_{t}^{0}\left(s_{t}\right)\right]$ solve problem (9), whose parameters are $\left(\alpha, \beta, \gamma, \lambda_{t}, n_{t}, s_{t}, p_{t+1}\right)$. If both goods are non-inferior, it follows at once that:

$$
\frac{\partial e_{t}^{0}\left(s_{t}\right)}{\partial \Lambda_{t}\left(s_{t}\right)} \geq 0, \frac{\partial c_{t}^{0}\left(s_{t}\right)}{\partial \Lambda_{t}\left(s_{t}\right)} \geq 0
$$

Inspection of eq. (8) reveals that an increase in $p_{t+1}$ induces an increase in $e_{t}^{0}\left(s_{t}\right)$ if $e_{t}^{0}\left(s_{t}\right) \in$ $(0,1)$, and preserves $e_{t}^{0}\left(s_{t}\right)=1$; for it increases the weight on $v\left(\lambda_{t+1}\right)$ relative to that on $u\left(c_{t}\left(s_{t}\right)\right)$. An increase in $p_{t+1}$ therefore has the opposite effect on $c_{t}^{0}\left(s_{t}\right)$.

The remaining comparative static results concern the effect of family status in the present on investment in, and the accumulation of, human capital. Note that the upper boundaries of the budget sets in the case $s_{t}=2$ lie strictly inside that associated with $s_{t}=1$ and that the price of $c_{t}$ relative to $e_{t}$ is lower for $s_{t}=2$ than for $s_{t}=1$. We then obtain $e_{t}^{0}(1) \geq e_{t}^{0}(2)=e_{t}^{0}(3)$.

To close this account of the household's behaviour, we introduce the central assumption that altruism is not operative when the adults are insufficiently educated, i.e., there exist some $\Delta>0$ such that $e_{t}^{0}(1)=0$ for all $\Lambda_{t}(1) \leq 2+\Delta$ and for all admissible $p_{t+1}$. This also yields $e_{t}^{0}(2)=e_{t}^{0}(3)=0$ as a trivial corollary.

\subsubsection{Dynamics}

Recalling that $e_{t}^{0}\left(s_{t}\right)$ is chosen so as to solve problem (9), eq. (2) may be written

$$
\lambda_{t+1}= \begin{cases}z\left(s_{t}\right) f\left(e_{t}^{0}\left(\Lambda_{t}\left(s_{t}\right), s_{t}, p_{t+1}\right)\right) \Lambda_{t}\left(s_{t}\right)+1, & s_{t}=1,2,3 \\ 1, & s_{t}=4 .\end{cases}
$$

Eq. (10) describes a random dynamical system, in the sense that although each child attains $\lambda_{t+1}$ in adulthood with certainty, he or she can wind up in any of the states $s_{t+1} \in\{1,2,3,4\}$ after reaching adulthood and forming a family. We assume henceforth that $2 z(1) f(1) \geq 1$, so that unbounded growth in the absence of premature mortality is possible (Bell and Gersbach, 
2001). The ensuing heterogeneity and its consequences for the system as a whole are quite complicated, but it will suffice from a policy perspective to focus on critical stationary states, which will now be introduced.

\subsection{Mortality Shocks and Economic Collapse}

The process by which the outbreak of an epidemic can lead to an economic collapse can be understood by examining an idealised initial state. At the start of period $t=0$, a society of homogeneous two-parent families, each with adult human capital endowment $2 \lambda_{0}$, is suddenly assailed by some fatal disease that afflicts young adults. Immediately after their children are born, they learn whether they are infected, and the survivors then choose $\left(c_{0}^{0}\left(\Lambda_{0}\left(s_{0}\right), s_{0}\right), e_{0}^{0}\left(\Lambda_{0}\left(s_{0}\right), s_{0}\right)\right)$ for $s_{0}=1,2,3$. Children who are left as unsupported orphans $\left(s_{0}=4\right)$ fall into the poverty trap at once. The assumption that altruism is not operative for $\Lambda_{t}=2$ also implies that $e_{t}^{0}(2,1)=0 \forall t$ : even if both parents survive but have been orphans in childhood, they cannot afford to send their children to school. In the absence of support, therefore, all orphans fall into the poverty trap, and their succeeding lineage remains there.

In order to discover what happens to the rest, we introduce the critical value function $\lambda^{*}(s, p)$ for stationary values of fertility and premature mortality: $n_{t}=n, \forall t$ and $p_{t}=p, \forall t$. This is defined by:

$$
\lambda^{*}(s, p)=z(s) f\left(e^{0}\left(\Lambda^{*}(s, p), s, p\right)\right) \Lambda^{*}(s, p)+1, \quad s=1,2,3
$$

where $\Lambda^{*}(1, p)=2 \lambda^{*}(1, p), \Lambda^{*}(2, p)=\Lambda^{*}(3, p)=\lambda^{*}(2, p)=\lambda^{*}(3, p) . \quad \lambda^{*}(s, p)$ is the stationary level of human capital associated with a particular state $s$, that is, in any pair of generations, parent(s) and offspring share the same level of human capital when both share the same state. It is clear from the above assumptions about altruism that $\lambda^{*}(s, p)=1$ satisfies (11). The assumption that $2 z(1) f(1) \geq 1$ also ensures, however, that there is at least one value of $\lambda^{*}(s, p)$ that exceeds unity if $p$ is not too small. ${ }^{4}$ Let $\lambda^{*}(s, p)$ denote the small-

\footnotetext{
${ }^{4}$ This claim follows by continuity from the associated proposition in Bell and Gersbach (2001), in which there is no premature adult mortality.
} 
est such value. Then, if a family is in state $s$, we have the following relationship between the parents' human capital and that attained by their children in adulthood when the shock represented by $p<1$ is permanent:

$$
\lambda_{t+1} \gtreqless \lambda_{t} \quad \text { according as } \quad \lambda_{t} \gtreqless \lambda^{*}(s, p) \text {. }
$$

Hence, there is a poverty trap. For if human capital does start to decrease, it will eventually reach $\lambda=1,{ }^{5}$ which is a stable stationary state.

An increase in premature adult mortality increases $\lambda^{*}(s, p), s=1,2,3$. To be precise, we have $\partial \lambda^{*}(s, p) / \partial p<0, s=1,2,3$ and $\lambda^{*}(1, p) \leq \lambda^{*}(2, p)=\lambda^{*}(3, p)$ (Bell, Devarajan and Gersbach, 2003). The first statement implies that an increase in premature adult mortality may cause a group that was earlier enjoying self-sustaining growth to fall into the poverty trap. The second implies that single-parent families need higher individual levels of human capital than two-parent families in order to escape the trap, so that an increase in premature adult mortality also increases the share of each generation that falls into the poverty trap by increasing the proportion of one-parent families.

A society that is assailed by such a disease over a long enough stretch of time and that is unable to make satisfactory arrangments for the care and support of orphans will descend progressively into the poverty trap. The sources of this collapse are threefold. First, if both parents die early, the transmission of knowledge across generations will be interrupted and the orphans will end up with minimal human capital. ${ }^{6}$ Second, the loss of income due to disability and early death reduces the lifetime resources available to the family, which may well result in the children spending much less time (if any at all) at school. Finally, the chance that the children themselves will contract the disease in adulthood makes investment in their education less attractive, even when both parents themselves remain uninfected.

\footnotetext{
${ }^{5}$ Strictly speaking, this statement holds when the system starts with $s_{t}=1$ or if $s_{t}=2,3$ and the system remains in those states for some time.

${ }^{6}$ Note that this effect alone causes a collapse in the long term.
} 


\section{The Policy Problem}

\subsection{Rationale and Instruments}

In the light of section 2, there is a clear and compelling rationale for public intervention, namely, to stave off possible economic collapse, with all of its baneful social and human consequences, which an epidemic like AIDS threatens to set in train. In order to draw up a plan of action, it is important to identify the main reasons why policy intervention is desirable in this setting. They involve different types of externalities. The first arises when the improvements in all future generations' welfare that would stem from a better education of today's children and from their good health in adulthood are not fully reflected in the preferences of today's parents, who are assumed to make the relevant decisions in the present. If, as is arguable, the government has - or should have - a longer horizon than individual households, then the case for intervention to promote schooling at the expense of child labor and to lower premature adult mortality by combatting the disease is, in principle, established. The second arises from the fact that communicable diseases have the character of a common property resource. The argument for intervention is reinforced when the disease reduces the returns to investment in human capital. Taken together, these arguments provide a strong justification for governments to promote education and to combat diseases, especially the communicable kind like AIDS, and to do so in a way that makes future policy credible.

There are three kinds of instruments available to the government to attain the broad objective of self-sustaining growth in a disease-ridden environment: (i) subsidies designed to encourage education; (ii) spending on measures to combat the spread of the disease and to treat those infected by it; and (iii) raising the taxes needed to finance these expenditures. The associated policies are now discussed in greater detail. 


\subsubsection{Education Policy}

The direct promotion of education takes the form of subsidies to households. These are paid either as general transfers or, more efficiently, conditional on the children attending school. They are financed by taxes on income, where it should be noted that a household's ability to pay depends on its state. For simplicity, therefore, we assume that the government can identify both a household and its state and that only the income of (healthy) adults is taxable. ${ }^{7}$ In using the preceding model to analyse alternative policies, we assume constant fertility at the level $n_{0}$ in the first two generations. Hence, $N_{0}=1, N_{1}=n_{0} / 2$ and $N_{2}=\left(n_{0} / 2\right)^{2}$.

Let $\tau_{t}\left(\Lambda_{t}^{i}\left(s_{t}\right)\right)$ denote the tax levied on household $i$ in period $t$ if it is in state $s_{t}$. Some fraction of the population will be subsidized out of the ensuing revenues. Starting with general transfers, we denote by $g_{t}^{i}\left(\Lambda_{t}^{i}\left(s_{t}\right)\right)$ the subsidy household $i$ will receive in period $t$ in state $s_{t}$, where subsidies should be interpreted in a broad sense; for instance, they may take the form of local infrastructure to support production.

Although the family chooses $e_{t}^{i}$ on the basis of its potential full income after tax, it is important to note that an increase in $\lambda_{t}$ not only enlarges the feasible set in the space of $\left(c_{t}, \lambda_{t+1}\right)$, but also makes its upper boundary steeper. A decrease in the net tax burden, however, will simply shift the said boundary to the right. Note also that subsidization can be made dependent on income and the household's identity, i.e., on the index $i$. The household's optimal educational choice is therefore written as $e_{t}^{i 0}\left(\Lambda_{t}^{i}\left(s_{t}\right), \tau_{t}\left(\Lambda_{t}^{i}\left(s_{t}\right)\right)-g_{t}^{i}\left(\Lambda_{t}^{i}\left(s_{t}\right)\right), s_{t}, p_{t+1}\right)$.

If, instead, subsidies are payable only on condition of school attendance, then household $i$ 's budget constraint becomes

$$
\left[\left(3-s_{t}\right)+n_{t} \beta\right] c_{t}^{i}+n_{t} \cdot\left[\alpha \gamma-\sigma_{t}^{i}\left(\Lambda_{t}^{i}\left(s_{t}\right)\right)\right] e_{t}^{i} \leq \alpha\left[\left(3-s_{t}\right) \lambda_{t}^{i}+n_{t} \gamma\right], \quad s_{t}=1,2
$$

where $\sigma_{t}^{i}\left(\Lambda_{t}^{i}\left(s_{t}\right)\right)$ is the subsidy payable to the family for each unit of time each child spends at school. Recall from (2) that the states $s_{t}=2$ and $s_{t}=3$ are identical in this respect.

\footnotetext{
${ }^{7}$ Taxing only adults may be justified by the ease of tax evasion for child income. It is unlikely that allowing household income to be taxable would change the main results of the paper.
} 
Where ability to pay is concerned, we assume that there is a subsistence level $c^{\text {sub }}$ for each adult and $\beta c^{s u b}$ for each child which must be ensured under all circumstances. Allowing for the possibility that not all households with the same characteristics will receive subsidies, household $i$ 's tax burden is therefore constrained by:

$$
\alpha\left[\left(3-s_{t}\right) \lambda_{t}^{i}+n_{t} \gamma\right]-\tau_{t}\left(\Lambda_{t}^{i}\left(s_{t}\right)\right) \geq\left[\left(3-s_{t}\right)+n_{t} \beta\right] c^{s u b}, \quad s_{t}=1,2
$$

where it is assumed that all children work full-time when $\lambda_{t}^{i}=1$ and the household receives no subsidies.

In order to enable an escape from what we have termed a general state of economic backwardness without outside help, we allow for some limited ability to pay whenever $\lambda_{t}^{i}=1$. In particular, the tax schedule for single-parent households must fulfill the condition

$$
0 \leq \tau_{t}(1) \leq \alpha\left(1+n_{t} \gamma\right)-\left(1+n_{t} \beta\right) c^{s u b} \equiv \tau^{b a}
$$

where it is plausible that $\tau^{b a}$ is small.

Turning to the formulation of educational policy, we denote by $\Lambda^{a}(s, p)$ the lowest level of a family's adult human capital in state $s$ such that full education will be chosen for the children in the absence of any government intervention. $\lambda^{a}(s, p)$ denotes the corresponding adult's human capital, with $\Lambda^{a}(1, p)=2 \lambda^{a}(1, p)$, and $\Lambda^{a}(s, p)=\lambda^{a}(s, p)$ for $s=2,3$.

We make two assumptions regarding education policies. We assume that it is possible to subsidize uneducated parents such that their children overshoot $\lambda^{a}(1,1)$ by enough to enable them to pay $\tau^{b a}$ in taxes if premature mortality is expected to be wholly prevented, i.e. if $p=1$. For this to hold, the educational technology must be sufficiently productive.

\section{Assumption 1}

There exists a positive, bounded subsidy $\bar{g}$ such that

$$
z(2) f\left(e^{0}\left(1, \tau^{b a}-\bar{g}, 2,1\right)\right) \cdot 1+1=\lambda^{a}(1,1)+\tau^{b a} /(2 \alpha)
$$

Since it is the net income of the adults, $\alpha+\bar{g}-\tau^{b a}$, that matters for the choice of education, $e^{0}$ is now written as a function of net income. Assumption 1 states that the combination 
of the educational technology and the transmission factor $z(2)$ is strong enough to yield a bounded transfer $\bar{g}$ (gross of the tax $\tau^{b a}$ ) that will induce a single-parent family whose adult human capital is unity to choose an $e^{0} \in(0,1]$ such that its children will attain the level of human capital $\lambda^{a}(1,1)+\tau^{b a} /(2 \alpha){ }^{8}$ Note that $\bar{g}$ is a gross subsidy, since the single-parent family pays taxes $\tau^{b a}$.

The assumption implies that two children whose parents have received $\bar{g}$ could pay at least $\tau^{b a}$, where they will form a two-parent family in adulthood while also choosing to educate their own children fully.

Secondly, we appeal to the existence of satisfactory institutional arrangements for the care and education of full orphans, whereby the cost per child may be larger than that corresponding to the family transfer $\bar{g}$. The following assumption allows, in principle, a solution to this social problem if fiscal resources are large enough over some sequence of periods.

\section{Assumption 2}

By using a sufficient amount of resources, a full orphan can be supported in such a way that he/she will attain a level of human capital of at least $\lambda^{a}(1,1)$ as an adult.

In order to complete the preliminaries, we introduce some additional notation. Let $\delta_{t} \in[0,1]$ denote the share of all $N_{t}$ families that receive the gross subsidy $\bar{g}$ in period $t$.

\subsubsection{Health Policy}

Health policy takes the form of spending on measures to combat the disease. Here we distinguish not only between prevention and treatment, but also between expenditures that produce private and public goods. For some diseases, treatment may result in a complete cure. There is no such prospect for the victims of AIDS; but the treatment of opportunistic infections in the later stages and the use of highly active anti-retroviral therapies (HAART) can pro-

\footnotetext{
${ }^{8}$ Our reference value of human capital is $\lambda^{a}$ where there is full schooling. In principle, our procedure can be applied to other human capital levels above $\lambda^{*}$ and lower than $\lambda^{a}$. The qualitative insights in this paper are not affected by the reference point.
} 
long life and maintain productivity. In the present OLG setting, therefore, treatment may be thought of as reducing premature adult mortality in the probabilistic sense. The distinction between private and public goods is also an idealization, and a hard one to draw where communicable diseases are concerned. Regular exercise and a good diet, for example, will lower the chances of heart disease only among those individuals who take this prescription seriously. Staying at home when suffering from influenza, however, reduces one's chances of passing on infection to others. Large-scale public programs aimed at combatting the spread of communicable diseases yield benefits for all, and large externalities are present. Examples are public awareness campaigns and, in the case of AIDS, the provision of condoms at no charge. The public good nature of such health policies stems from the positive externalities that arise when other individuals benefit from the efforts of an individual to lower his or her risk of getting infected, or, if already infected, the risk of also infecting others.

Formulating the effects of health policy is fairly straightforward in the 'pure' cases, on which we focus. Let the probability that an adult in family $i$ born in period $t-1$ will survive into old age in period $t$ be denoted by $p_{t}^{i}$. If spending on prevention, broadly construed, produces a purely private good for the individual in question, then

$$
p_{t}^{i}=p\left(h_{t}^{i}, \Lambda_{t}^{i}\right)
$$

where $h_{t}^{i}$ is the amount spent on the individual in question. We specialize $p\left(h_{t}^{i}, \cdot\right)$ as follows.

\section{Assumption 3}

If spending on health produces a purely private good, then

$$
p\left(h_{t}^{i}, \cdot\right)= \begin{cases}1 & \text { if } h_{t}^{i} \geq \bar{h} \\ p^{p r} & \text { otherwise }\end{cases}
$$

The constant $\left(p^{p r}<1\right)$ is the probability that an adult male or female will survive to old age if spending on him or her should fall below $\bar{h}$. Allowing spending in the amount $\bar{h}$ to eliminate the risk of premature mortality altogether is a convenient simplification.

An assumption about the incidence of the disease within the population is also needed. 


\section{Assumption 4}

Premature mortality is independently and identically distributed across individuals. Hence, in the case of health production as a purely private good, we have

$$
[\pi(1), \pi(2), \pi(3), \pi(4)]=\left[\left(p^{p r}\right)^{2}, p^{p r}\left(1-p^{p r}\right), p^{p r}\left(1-p^{p r}\right),\left(1-p^{p r}\right)^{2}\right]
$$

At the other extreme, let spending on prevention produce a pure public good, where it is quite possible that the size of the population may affect the efficacy of spending. Hence, we may write $p_{t}=p\left(\eta_{t}, N_{t}\right)$, where the measure of families in period $t$ is $N_{t}$, and $\eta_{t}$ denotes the level of spending per family. Recall that the measure of all families at the start of period 0 has been normalized to unity. Analogously to Assumption 3, we have

\section{Assumption 5}

Spending at least the aggregate amount $\bar{\eta} N_{t}$ on public health in period $t$ eliminates all premature mortality in that period; spending less has no effect and the level is exogenously given. That is,

$$
p\left(\eta_{t}, \cdot\right)= \begin{cases}1 & \text { if } \eta_{t} \geq \bar{\eta} \\ p^{p u} & \text { otherwise }\end{cases}
$$

Of course, in most African countries eliminating all premature mortality is not possible. The present assumption merely simplifies the exposition. At the cost of more notation and technical assumptions, our results can be extended to the case when public health reduces premature mortality to a certain extent.

As indicated above, the treatment of diseases prolongs productive life, even if no cure is available. When the disease is also communicable, substantial external benefits will often result. In the framework adopted here, the ensuing private and external benefits will be represented as stemming from a reduction in premature adult mortality. 


\subsection{The Efficacy of a Single Policy Instrument}

We now turn to policy choices, whereby trade-offs depend, of course, on the objectives. Three different objectives are important: fostering the growth of income through education, combatting the spread of the disease, and treating those infected by it. We concentrate wholly on the first two, which we subsume under the single objective of maximizing the size of the tax base, which is largely provided by healthy, educated adults, at some date in the future. For this is vital to ensuring sustainable, long-term growth. In what follows, the future date is taken to be two generations after the outbreak $(t=2)$ as this will be sufficient to identify the relative performance of different policy approaches.

We assume that the initial configuration is one of a society in a state of general economic backwardness $\left(\lambda_{0}=1\right)$. This choice renders irrelevant what level of mortality happened to rule before period 0 .

It is useful to discuss briefly the consequences when the government intervenes in only one domain. Beginning with health policy, it is clear this alone does not foster education when parents are themselves uneducated, and thus will not promote human capital formation and income growth when all are uneducated. Hence, it will not be optimal to use health policy in isolation in period 0 .

Turning to education policy, there are conditions under which concentrating resources on the promotion of education can yield sustainable growth of human capital at an asymptotically steady rate. ${ }^{9}$ However, as we will see in the following, it is generally the case that allocating at least some resources to promoting health is advantageous.

\footnotetext{
${ }^{9} \mathrm{~A}$ sufficient condition for the attainment of universal full education within a finite number of periods is $(1-\pi(4)) z(2) f(1)>1$. Details of the lengthy calculations are available upon request.
} 


\section{Double- versus Single-Domain Targeting}

In this section we investigate how fiscal resources should be allocated between education and health, and how these resources should be concentrated on subgroups of the population when both types of policies can be deployed. We treat the cases of private and public goods separately.

We concentrate on the following comparison of DT and ST when the society is initially in a state of economic backwardness. In period $t=0$, one of the two schemes is put into effect. Under DT, supported families will receive both the benefits of spending on health and transfers to promote education. Under ST, selected families will receive only educational support in the first phase, but on reaching adulthood, their children will obtain the benefits of specific health policies. In period $t=1$, the scheme ST is completed for those families targeted in $t=0$, whereas DT is applied to newly targeted families in the current generation under both schemes. In all subsequent periods, we assume that an optimal scheme is used, but without specifying precisely how it operates. This allows us to concentrate on the comparison of DT and ST over the first two periods.

\subsection{Health Spending Produces a Private Good}

Since the most general case is extremely complex, we confine ourselves to representative ones. Recalling Assumption 1 and $\Lambda_{0}(1)=2$, observe that if $\bar{g}$ is paid to a two-parent family in period 0 , then its children will attain

$$
z(1) f\left(e^{0}\left(2, \tau^{b a}-\bar{g}, 1,1\right)\right) \cdot 2+1 \geq z(2) f\left(e^{0}\left(1, \tau^{b a}-\bar{g}, 2,1\right)\right) \cdot 1+1=\lambda^{a}(1,1)+\frac{\tau^{b a}}{2 \alpha} .
$$

Denote by $\bar{\tau}$ the largest amount of tax that a two-parent household formed by such children on reaching adulthood could pay, while still choosing $e^{0}=1$ if they expect that their children will be protected against premature mortality. It is clear that $\bar{\tau} \geq \tau^{b a}$, where the equality holds if, and only if, $z(1)=z(2) / 2$ and $e^{0}\left(1, \tau^{b a}-\bar{g}, 2,1\right)=1$. 
We assume that all families are taxed in period $t=0$, but only educated households in $t=1$.

The following proposition states a sufficient condition for ranking the targeting schemes:

\section{Proposition 1}

Suppose a society in a state of economic backwardness faces the permanent mortality rate $1-p^{p r}$ from $t=0$ onwards. Suppose also that Assumptions 1 to 4 hold and that $\bar{\tau}>2 \bar{h}$. Then, in the setting described above, DT is superior to ST if

$$
\frac{\bar{g}}{\bar{h}}>\frac{2 p^{p r}\left(2-p^{p r}\right)}{\left(1-p^{p r}\right)^{2}}
$$

The proof is given in the Appendix. Observe that condition (18) is necessary as well as sufficient for the superiority of DT if, and only if, both $z(1)=z(2) / 2$ and $e^{0}\left(1, \tau^{b a}-\bar{g}, 2,1\right)=$ 1 ; for only then do the children of single-parent families attain the same level of human capital as those reared by both parents, and only then does $\bar{\tau}=\tau^{b a}$ hold.

Proposition 1 has an intuitive interpretation. The higher the costs of promoting education relative to those of reducing premature adult mortality, the more attractive is DT, since it is relatively cheap to prevent the early death of adults educated in period zero. If, conversely, the costs of preventing premature mortality are relatively high, it is more efficient to promote education alone.

Establishing the effect of the severity of the shock on the ranking of the schemes is generally more involved. In the case $\bar{\tau}=\tau^{b a}$, however, we obtain a clear-cut result.

\section{Corollary 1}

If $\bar{\tau}=\tau^{b a}$, there exists a survival probability $\hat{p}^{p r} \in(0,1)$, implicitly given by

$$
\hat{p}^{p r}=1-\sqrt{1-\frac{\bar{g}\left(\hat{p}^{p r}\right)}{\bar{g}\left(\hat{p}^{p r}\right)+2 \bar{h}}},
$$

such that $D T$ is superior to $S T$ if, and only if, $p^{p r}<\hat{p}^{p r}$.

The proof of Corollary 1 is given in the Appendix. Corollary 1 implies that mortality above the threshold value $1-\hat{p}^{p r}$ makes DT more attractive relative to ST. The reason is that high mortality makes educational support alone less attractive, because many of the children who 
benefit will not survive early adulthood. If, however, the probability that adults will survive into old age is sufficiently high, then it is more effective to promote the formation of human capital in period 0 .

It is obvious that our criterion for judging DT and ST neglects other important considerations. In practice, two other criteria would surely come into play in the overall assessment. First, ST spreads support - be it in the realm of health, education or both - more more widely than DT, which necessarily concentrates all resources on fewer households. Second, DT lowers the number of terminally ill people in period 0 , and thus may be preferred on this ground alone.

\subsection{Health Spending Produces a Public Good}

When spending on health produces a public good, we also allow for aid from outside the system, as this may be important for the relative attractiveness of DT and ST. Hence, suppose that outside help in the amount of $\bar{B}_{0} \geq 0$ is available in period $t=0$. As before, we assume that all families are taxed in period $t=0$, but only educated households in $t=1$.

We then obtain:

\section{Proposition 2}

Suppose a society in a state of economic backwardness faces the permanent mortality rate $1-p^{p u}$ from $t=0$ onwards. Suppose also that total fiscal resources in period 0 , denoted by $B_{0}$, exceed $\bar{\eta}$, and that Assumptions 1,2, 3 and 5 hold. Then DT is superior to ST if

$$
B_{0} \equiv \tau^{b a}+\bar{B}_{0}>\frac{\bar{\eta}}{(1-p)^{2}}
$$

The proof is given in the Appendix.

In the absence of outside aid, condition 20 can be reduced further to $\left(1-p^{p u}\right)^{2} \tau^{b a}>\bar{\eta}$. Hence, DT is then superior to ST if and only if $\left(1-p^{p u}\right)^{2} \in\left(\frac{\bar{\eta}}{\tau^{b a}}, 1\right)$.

The intuition runs as follows: the more orphans the disease creates, the more attractive is 
DT; for under the above assumptions, DT eliminates the disease, and so preserves in period 1 all the investment in education in period 0 . Foreign aid in period 0 , when public funds are likely to be especially scarce, also works in favor of DT. This general conclusion provides a foundation for using health policy from the start in the formulation of good policy programs.

We consider once more the impact of the level of mortality on the relative attractiveness of DT and ST. We obtain, analogously to Corollary 1:

\section{Corollary 2}

$\mathrm{DT}$ is superior to ST if $p^{p u}<\tilde{p}^{p u} \equiv 1-\sqrt{\frac{\bar{\eta}}{\tau^{b a}+\overline{B_{0}}}}$.

The proof of Corollary 2 is given in the Appendix. Corollary 2 implies that high mortality makes DT more attractive relative to ST, as intuition would suggest. An important implication of Corollary 2 is that more outside aid also makes DT more attractive relative to ST.

\section{Conclusion}

The purpose of this paper has been to analyze what we have called the macroeconomics of targeting in the context of an epidemic disease like HIV/AIDS, which causes heavy mortality among young adults. We have focused on a specific setup, in which the economy is initially in a stationary state of backwardness, while making a variety of other, deliberately chosen, simplifying assumptions. Numerous issues deserve further inquiry. For instance, the character of optimal targeting schemes strongly depends on the form and strength of aversion to inequality. If aversion to inequality in all forms is so extreme that all needy families must be treated equally, then neither policies to combat the disease nor those to foster human capital formation can be successful in bringing about sustained growth, even when spending on health produces a pure public good. Yet as the model exhibits a strong and inherent trade-off between growth and inequality in both domains, the awkward choices it poses can only be resolved within the society in question itself.

The solution to the targeting problem may have a quite different character when a growing 
economy is hit by an epidemic shock. In such cases, single targeting with respect to health may be optimal, as it protects the lives of educated adults in the immediate aftermath as well as the future returns from human capital formation in their children. Finally, we emphasize once more the illustrative purpose of our simple model. It can, and should, be generalized to deal with more finely formulated aspects both of the epidemiological environment and of health and educational schemes, while paying careful attention to the empirical evidence in all areas. We hope that the present paper provides both a start in, and an impetus to, this line of research. 


\section{Appendix}

\subsection{Proof of Proposition 1}

We proceed by comparing double and single targeting. For convenience, we define $p:=p^{p r}$ and use $\lambda_{t}^{d_{t}}$ to denote the human capital of individuals in particular groups, indexed by $d_{t}$ $\left(d_{t}=1,2, \ldots, D_{t}\right)$, in period $t$, whereby the nature and number of groups, $D_{t}$, may vary across time depending on the interventions employed.

\section{Double Targeting}

In period $t=0$, when $N_{0}=1$, let each of a group of families simultaneously receive health and education support of $2 \bar{h}$ and $\bar{g}$, respectively, so that all the adults in question will survive to old age. The share of all families that can be supported in this way is given by

$$
\delta_{0}=\frac{B_{0}}{\bar{g}+2 \bar{h}}
$$

where $B_{0}=\tau^{b a}$. In period $t=0$, therefore, we obtain the following societal pattern:

- $\delta_{0} n_{0}$ children reach, as adults, $\lambda_{1}^{1}=z(1) f\left(e^{0}\left(2, \tau^{b a}-\bar{g}, 1,1\right)\right) \cdot 2+1$

- $\left(2 p-p^{2}\right)\left(1-\delta_{0}\right) n_{0}$ children go uneducated, and attain a human capital level of 1

- $(1-p)^{2}\left(1-\delta_{0}\right) n_{0}$ children are left as full orphans, who also attain unity

In period $t=1$, the educated children marry among themselves and form $\delta_{0} n_{0} / 2$ families, each of which has $n_{1}$ children who reach, as adults, $\lambda_{2}^{1}=z(1) f(1) \cdot 2 \lambda_{1}^{1}+1$ in period 2 . All such families can pay $\bar{\tau}$ in taxes, provided they continue to receive health support in the amount of $\bar{h}$ per adult. With the revenues $B_{1}=\delta_{0} n_{0} \bar{\tau} / 2$, the assumption that $\bar{\tau}>2 \bar{h}$ implies that the government can afford to subsidize a further share of families, denoted by $\delta_{1}$, in the same manner as in period 0 :

$$
n_{0} \delta_{0} \bar{\tau} / 2=\left[\delta_{0} 2 \bar{h}+\delta_{1}(\bar{g}+2 \bar{h})\right] n_{0} / 2
$$


or

$$
\delta_{1}=\frac{\delta_{0}(\bar{\tau}-2 \bar{h})}{\bar{g}+2 \bar{h}} .
$$

Hence, in period $t=1, \delta_{1}\left(n_{0} / 2\right)$ families receive subsidies, and their children reach, as adults, $\lambda_{2}^{2}=\lambda_{1}^{1}$ in period 2. At $t=2$, therefore, we have the following two groups of educated children:

- $\left(\delta_{0} n_{0} / 2\right) n_{1}$ individuals possess $\lambda_{2}^{1}=2 z(1) f(1) \lambda_{1}^{1}+1$

- $\left(\delta_{1} n_{0} / 2\right) n_{1}$ individuals possess $\lambda_{2}^{2}=\lambda_{1}^{1}$

We assume, as usual, that $2 z(1) f(1) \geq 1$. This implies that $\lambda_{2}^{1} \geq \lambda_{1}^{1}+1$. Hence, if families with $\Lambda_{1}=2 \lambda_{1}^{1}$ can pay $\bar{\tau}$ when protected against premature death, then those with $2 \lambda_{2}^{1}$ can pay at least $\bar{\tau}+2 \alpha$. To be rather more precise, they can pay $\bar{\tau}+2 \alpha+\zeta(1)$ where $\zeta(1)$ denotes the additional taxable capacity beyond $\bar{\tau}+2 \alpha$. Note that $\zeta(1)=0 \Longleftrightarrow 2 z(1) f(1)=1$. The total taxable capacity in period $t=2$ is therefore

$$
\frac{n_{0}}{2} \frac{n_{1}}{2}\left[\delta_{0}(\bar{\tau}+2 \alpha+\zeta(1))+\delta_{1} \bar{\tau}\right]=\frac{n_{0}}{2} \frac{n_{1}}{2}\left[\left(\delta_{0}+\delta_{1}\right) \bar{\tau}+\delta_{0}(2 \alpha+\zeta(1))\right]
$$

From period 2 onwards, we assume that the society operates under an optimal education and health support system, whose precise nature we do not need to specify.

\section{Single Targeting}

In period $t=0$, each of a group of families receives the transfer $\bar{g}$. The share of families supported in this way is given by:

$$
w_{0}=\frac{B_{0}}{\bar{g}} .
$$

This intervention generates the following societal structure:

- $p^{2} w_{0} n_{0}$ children reach $\lambda_{1}^{1}=z(1) f\left(e^{0}\left(2, \tau^{b a}-\bar{g}, 1,1\right)\right) \cdot 2+1$

- $2 p(1-p) w_{0} n_{0}$ children reach $\lambda_{1}^{2}=\lambda^{a}(1,1)+\frac{\tau^{b a}}{2 \alpha}$ 
- $(1-p)^{2} w_{0} n_{0}$ children are left as full orphans, who attain a human capital level of 1

- another $(1-p)^{2}\left(1-w_{0}\right) n_{0}$ children also become full orphans, attaining unity

- $\left(2 p-p^{2}\right)\left(1-w_{0}\right) n_{0}$ children have at least one parent, but receive no education, and so attain unity

In period $t=1$, the adults who were educated in period 0 can be taxed. Hence, tax revenues in period 1 will be

$$
B_{1}=\left[p^{2} \bar{\tau}+2 p(1-p) \tau^{b a}\right] \frac{w_{0} n_{0}}{2}
$$

It may be possible to subsidize a further share, $w_{1}$, of households, even while fully protecting those educated in period 0 against premature mortality in period 1 . The budget constraint is $B_{1} \geq\left(2 p-p^{2}\right) \bar{h} w_{0} n_{0}+(\bar{g}+2 \bar{h}) w_{1} n_{0} / 2$, which yields

$$
w_{1}=w_{0} \frac{2 p\left(\tau^{b a}-2 \bar{h}\right)-p^{2}\left(2 \tau^{b a}-2 \bar{h}-\bar{\tau}\right)}{\bar{g}+2 \bar{h}} .
$$

In period $t=1$, we have therefore under ST three goups of educated children

- $p^{2} w_{0}\left(n_{0} / 2\right) n_{1}$ children reach, as adults, $\lambda_{2}^{1}=2 z(1) f(1) \lambda_{1}^{1}+1$

- $2 p(1-p) w_{0}\left(n_{0} / 2\right) n_{1}$ children reach, as adults, $\lambda_{2}^{2}=z(1) f(1) \lambda_{1}^{2}+1$

- $w_{1}\left(n_{0} / 2\right)$ families receive subsidies, and their children reach, as adults, $\lambda_{2}^{3}=\lambda_{1}^{1}$.

Hence, the total taxable capacity is

$$
\frac{n_{0}}{2} \frac{n_{1}}{2}\left\{p^{2} w_{0}(\bar{\tau}+2 \alpha+\zeta(1))+2 p(1-p) w_{0}\left(\tau^{b a}+2 \alpha+\zeta(2)\right)+w_{1} \bar{\tau}\right\}
$$

where the term in the bracket can be rewritten as

$$
\left[\left(2 p-p^{2}\right) w_{0}+w_{1}\right] \bar{\tau}+\left(2 p-p^{2}\right) w_{0} 2 \alpha-2 p(1-p) w_{0}\left(\bar{\tau}-\tau^{b a}\right)+\left[2 p(1-p) \zeta(2)+p^{2} \zeta(1)\right] w_{0}
$$

The term $\zeta(2)$ denotes the additional taxable capacity beyond $\tau^{b a}+2 \alpha$. Note that $\zeta(2) \leq$ $\zeta(1)$ 
Note that the share of families newly subsidized in $t=1$ receive support in both domains and are therefore doubly targeted. This makes both systems comparable. ${ }^{10}$ From $t=2$ onwards, the society operates under the same optimal program as under DT.

\section{Comparison}

We begin by noting that $z(1) f\left(e^{0}\left(2, \tau^{b a}-\bar{g}, 1,1\right)\right) \cdot 2+1 \geq z(2) f\left(e^{0}\left(1, \tau^{b a}-\bar{g}, 2,1\right)\right) \cdot 1+1$. To compare DT and ST, it suffices to compare the total taxable capacities in period $t=2$, i.e. the expressions

$$
\begin{aligned}
A^{D} \equiv & \left(\delta_{0}+\delta_{1}\right) \bar{\tau}+\delta_{0}(2 \alpha+\zeta(1)) \\
A^{S} \equiv & {\left[\left(2 p-p^{2}\right) w_{0}+w_{1}\right] \bar{\tau}+w_{0}\left[\left(2 p-p^{2}\right) 2 \alpha+\left(p^{2} \zeta(1)+2 p(1-p) \zeta(2)\right)\right] } \\
& -w_{0}\left[2 p(1-p)\left(\bar{\tau}-\tau^{b a}\right)\right] .
\end{aligned}
$$

Note that

$$
\begin{array}{ll}
\zeta(1) \geq \zeta(2) \quad \text { with equality } & \Longleftrightarrow 2 z(1)=z(2) \wedge e^{0}\left(1, \tau^{b a}-\bar{g}, 2,1\right)=1 \\
\bar{\tau} \geq \tau^{b a} \quad \text { with equality } \Longleftrightarrow 2 z(1)=z(2) \wedge e^{0}\left(1, \tau^{b a}-\bar{g}, 2,1\right)=1 .
\end{array}
$$

Then, $A^{S} \leq\left[\left(2 p-p^{2}\right) w_{0}+w_{1}\right] \bar{\tau}+w_{0}\left(2 p-p^{2}\right)(2 \alpha+\zeta(1))$ with equality if and only if $2 z(1)=z(2)$ and $\quad e^{0}\left(1, \tau^{b a}-\bar{g}, 2,1\right)=1$. It follows that a sufficient condition for the superiority of DT is $A^{D}>\left[\left(2 p-p^{2}\right) w_{0}+w_{1}\right] \bar{\tau}+w_{0}\left(2 p-p^{2}\right)(2 \alpha+\zeta(1))$ or

$$
\left[\left(\boldsymbol{\delta}_{0}+\boldsymbol{\delta}_{1}\right)-\left(\left(2 p-p^{2}\right) w_{0}+w_{1}\right)\right] \bar{\tau}+\left(\boldsymbol{\delta}_{0}-\left(2 p-p^{2}\right) w_{0}\right)(2 \alpha+\zeta(1))>0
$$

From (21) and (23), we know that

$$
\begin{aligned}
\delta_{0}-\left(2 p-p^{2}\right) w_{0} & =B_{0}\left(\frac{1}{\bar{g}+2 \bar{h}}-\frac{p(2-p)}{\bar{g}}\right) \\
& =B_{0}\left(\frac{(1-p)^{2} \bar{g}-2 p(2-p) \bar{h}}{\bar{g}(\bar{g}+2 \bar{h})}\right)
\end{aligned}
$$

It follows that $\delta_{0}-\left(2 p-p^{2}\right) w_{0}>0$ if and only if

$$
\frac{\bar{g}}{\bar{h}}>\frac{2 p(2-p)}{(1-p)^{2}}
$$

\footnotetext{
${ }^{10}$ Essentially, we compare a sequence of education and health subsidies with simultaneous subsidies over one generation.
} 
From (22) - (24), some manipulation reveals that $\left(\delta_{0}+\delta_{1}\right)-\left(\left(2 p-p^{2}\right) w_{0}+w_{1}\right)>0$ is equivalent to

$$
\frac{\bar{g}}{\bar{g}+2 \bar{h}} \cdot\left(1+\frac{\bar{\tau}-2 \bar{h}}{\bar{g}+2 \bar{h}}\right)>\left(2 p-p^{2}\right) \cdot\left[\left(1+\frac{\tau^{b a}-2 \bar{h}}{\bar{g}+2 \bar{h}}\right)+\frac{p^{2}}{2 p-p^{2}} \cdot \frac{\bar{\tau}-\tau^{b a}}{\bar{g}+2 \bar{h}}\right]
$$

Now the expression in brackets on the LHS of this inequality is greater than that in brackets on the RHS for all $p \in(0,1)$ if $\bar{\tau}>\tau^{b a}$; the expressions are equal if $\bar{\tau}=\tau^{b a}$. It follows that the inequality holds if $\bar{g} /(\bar{g}+2 \bar{h})>p(2-p)$ or, equivalently,

$$
\frac{\bar{g}}{\bar{h}}>\frac{2 p(2-p)}{(1-p)^{2}} \text {. }
$$

Hence, if (25) holds, DT is superior over ST. Observe that if $\bar{\tau}=\tau^{b a}$, then the said condition (25) is also necessary for the superiority of DT.

\section{Proof of Corollary 1:}

From proposition $1, \bar{\tau}=\tau^{b a}$ implies that $\frac{\bar{g}\left(p^{p r}\right)}{\bar{h}}>\frac{4 p^{p r}-2\left(p^{p r}\right)^{2}}{\left(1-p^{p r}\right)^{2}}$ is necessary and sufficient for the superiority of DT over ST. We therefore solve $\frac{\bar{g}\left(p^{p r}\right)}{\bar{h}}=\frac{4 p^{p r}-2\left(p^{p r}\right)^{2}}{\left(1-p^{p r}\right)^{2}}$ for the survival probability and obtain

$$
\left(p^{p r}\right)^{2}-2 p^{p r}+\frac{\bar{g}\left(p^{p r}\right)}{\bar{g}\left(p^{p r}\right)+2 \bar{h}}=0
$$

which implies

$$
p^{p r}=1 \pm \sqrt{1-\frac{\bar{g}\left(p^{p r}\right)}{\bar{g}\left(p^{p r}\right)+2 \bar{h}}} .
$$

Since the solution must lie in $[0,1]$, only the second solution with the negative sign is relevant. The RHS is strictly monotonically decreasing with $p^{p r}$ for this solution. For $p^{p r}=0(1)$, the LHS is smaller (larger) than the RHS. This establishes the uniqueness of $\hat{p}^{p r}$. 


\subsection{Proof of Proposition 2}

Again, for notational convenience we set $p:=p^{p u}$ in this proof. We use the same line of reasoning employed in the proof of Proposition 1. Under DT with health spending $\bar{\eta}$, there will be no premature adult mortality, and the share of all families that can be given a transfer in the amount $\bar{g}$ in period 0 is

$$
\delta_{0}=\frac{B_{0}-\bar{\eta}}{\bar{g}}
$$

where $B_{0} \geq \tau^{b a}$ as all families are taxed in period $t=0$ and, by hypothesis, $B_{0} \geq \bar{\eta}$. Total revenue in period 1 is then

$$
B_{1}=\delta_{0} \bar{\tau} N_{1}
$$

with $N_{1}=\frac{n_{0}}{2}$ so that the share of all families that can be given a transfer in the amount $\bar{g}$ in period 1 is

$$
\delta_{1}=\left[\delta_{0} \bar{\tau}-\bar{\eta}\right] / \bar{g}
$$

As in proposition 1 , the total taxable capability in period $t=2$ is

$$
\frac{n_{0}}{2} \frac{n_{1}}{2}\left[\delta_{0}(\bar{\tau}+2 \alpha+\zeta(1))+\delta_{1} \bar{\tau}\right]=\frac{n_{0}}{2} \frac{n_{1}}{2}\left[\left(\delta_{0}+\delta_{1}\right) \bar{\tau}+\delta_{0}(2 \alpha+\zeta(1))\right] .
$$

Under ST, we have the state vector $[\pi(1), \pi(2), \pi(3), \pi(4)]=\left[p^{2}, p(1-p), p(1-p),(1-p)^{2}\right]$ in period 0 . The share of all families that can be given a transfer in the amount $\bar{g}$ in period 0 is

$$
w_{0}=B_{0} / \bar{g}
$$

Note that $w_{0}=\delta_{0}+\frac{\bar{\eta}}{\bar{g}}$. This policy yields the following structure of human capital levels in period 1:

- $p^{2} w_{0} n_{0}$ children attain $z(1) f\left(e^{0}\left(2, \tau^{b a}-\bar{g}, 1,1\right)\right) \cdot 2+1$ and can pay $\frac{\bar{\tau}}{2}$ in $t+1$;

- $2 p(1-p) w_{0} n_{0}$ children attain $\lambda^{a}(1,1)+\frac{\tau^{b a}}{2 \alpha}$;

- $\left(2 p-p^{2}\right)\left(1-w_{0}\right) n_{0}$ have been raised by at least one parent and attain unity;

- $(1-p)^{2} n_{0}$ are left as full orphans, and attain 1 . 
Suppose $\bar{\eta} N_{1}$ is affordable. Then the gross tax revenue in period 1 will be

$$
\left[p^{2} w_{0} \bar{\tau}+2 p(1-p) w_{0} \tau^{b a}\right] N_{1}
$$

so that $\bar{\eta} N_{1}$ is indeed affordable if, and only if,

$$
\bar{\eta} \leq p^{2} w_{0} \bar{\tau}+2 p(1-p) w_{0} \tau^{b a}
$$

Since $w_{0}=B_{0} / \bar{g}$, some manipulation yields the equivalent condition.

$$
B_{0} \geq \frac{\bar{\eta} \bar{g}}{p^{2} \bar{\tau}+2 p(1-p) \tau^{b a}}
$$

If this condition is violated, however, that which we have called ST is, in fact, infeasible. From now on we assume that the condition holds as otherwise DT is superior to ST as ST is infeasible.

Analogously to $\delta_{1}$, we have

$$
w_{1}=\left[p^{2} w_{0} \bar{\tau}+2 p(1-p) w_{0} \tau^{b a}-\bar{\eta}\right] / \bar{g}
$$

As in proposition 1 , the total taxable capability in period $t=2$ is

$$
\frac{n_{0}}{2} \frac{n_{1}}{2}\left\{p^{2} w_{0}(\bar{\tau}+2 \alpha+\zeta(1))+2 p(1-p) w_{0}\left(\tau^{b a}+2 \alpha+\zeta(2)\right)+w_{1} \bar{\tau}\right\}
$$

Now recall that

$$
z(1) f\left(e^{0}\left(2, \tau^{b a}-\bar{g}, 1,1\right)\right) \cdot 2+1 \geq z(2) f\left(e^{0}\left(1, \tau^{b a}-\bar{g}, 2,1\right)\right)+1
$$

Hence, in order to establish the superiority of DT over ST, it suffices to compare the total taxable capacities in period $t=2$, and as in proposition 1 , a sufficient condition for the superiority of DT is

$$
\left[\left(\boldsymbol{\delta}_{0}+\boldsymbol{\delta}_{1}\right)-\left(\left(2 p-p^{2}\right) w_{0}+w_{1}\right)\right] \bar{\tau}+\left(\delta_{0}-\left(2 p-p^{2}\right) w_{0}\right)(2 \alpha+\zeta(1))>0
$$


Combining the expressions derived above, we have

$$
\delta_{0}+\delta_{1}>\left(2 p-p^{2}\right) w_{0}+w_{1}
$$

if, and only if,

$$
\frac{B_{0}-\bar{\eta}}{\bar{g}}+\frac{B_{0}-\bar{\eta}}{\bar{g}} \frac{\bar{\tau}}{\bar{g}}-\frac{\bar{\eta}}{\bar{g}}>\left(2 p-p^{2}\right) \frac{B_{0}}{\bar{g}}+\frac{p^{2} \bar{\tau}+2 p(1-p) \tau^{b a}}{\bar{g}} \frac{B_{0}}{\bar{g}}-\frac{\bar{\eta}}{\bar{g}} .
$$

Some tedious manipulation reveals that this condition can be written as

$$
B_{0}>\frac{\bar{\eta}[\bar{g}+\bar{\tau}]}{(1-p)^{2} \bar{g}+\left(1-p^{2}\right) \bar{\tau}-2 p(1-p) \tau^{b a}},
$$

and since $\bar{\tau} \geq \tau^{b a}$, it follows that

$$
\frac{\bar{\eta}[\bar{g}+\bar{\tau}]}{(1-p)^{2} \bar{g}+\left(1-p^{2}\right) \bar{\tau}-2 p(1-p) \tau^{b a}} \leq \frac{\bar{\eta}}{(1-p)^{2}}
$$

On the other hand, we have $\delta_{0}-\left(2 p-p^{2}\right) w_{0}>0$ if, and only if

$$
B_{0}>\frac{\bar{\eta}}{(1-p)^{2}}
$$

so if (27) is fullfilled, the condition (26) holds automatically. This establishes the result.

\section{Proof of Corollary 2:}

The condition $\tau^{b a}+\bar{B}_{0}=\frac{\bar{\eta}}{\left(1-p^{p u}\right)^{2}}$ implies

$$
\begin{gathered}
\left(p^{p u}\right)^{2}-2\left(p^{p u}\right)+1-\frac{\bar{\eta}}{\tau^{b a}+\bar{B}_{0}}=0 \\
p_{1 / 2}^{p u}=1 \pm \sqrt{\frac{\bar{\eta}}{\tau^{b a}+\bar{B}_{0}}}
\end{gathered}
$$

The only feasible solution is $\tilde{p}^{p u}=1-\sqrt{\frac{\bar{\eta}}{\tau^{b a}+\bar{B}_{0}}}$ and DT is superior to ST if $p^{p u}<\tilde{p}^{p u}$. 


\section{References}

[1] Bell, C., and Gersbach, H. (2001), 'Child Labor and the Education of a Society', IZA Discussion Paper No. 338, Bonn.

[2] Bell, C., Devarajan, S., and Gersbach, H. (2003), 'The Long-run Economic Costs of AIDS: Theory and an Application to South Africa', Policy Research Working Paper No. 3152, World Bank.

[3] Bell, C., Devarajan, S., and Gersbach, H. (2006), 'The Long-run Economic Costs of AIDS: A Model with an Application to South Africa', The World Bank Economic Review, forthcoming.

[4] Bruhns, R. (2004), ‘Child Labor and Fertility', mimeo, University of Heidelberg.

[5] Corrigan, P., Glomm, G., and Méndez, F. (2004), 'AIDS, Human Capital and Growth', mimeo.

[6] Corrigan, P., Glomm, G., and Méndez, F. (2005), 'AIDS Crisis and Growth', Journal of Development Economics, 77, pp. 107-124.

[7] Galasso, E., Ravallion, M. (2005), 'Decentralized targeting of an antipoverty program', Journal of Public Economics, 89, 705-727.

[8] Haacker, M., (ed.) (2004), The Macroeconomics of HIV/AIDS, Washington, DC: International Monetary Fund, pp. xvii +344 .

[9] McDonald, S., and Roberts, J. (2004), 'AIDS and Economic Growth: A Human Capital Approach', Sheffield Economic Research Paper Series, SERP Number: 2004008.

[10] Young, A. (2005), 'The Gift of the Dying: The Tragedy of AIDS and the Welfare of Future African Generations', Quarterly Journal of Economics, 120 (2). 